detail. These two schemes-one, the homopolar or bond eigenfunction method associated with the names of Heitler, London, Slater and Pauling ; the other, the method of molecular orbitals developed by Lennard-Jones, Hund and Mullikenalready provide a means of describing and explaining the valence behaviour of large groups of chemical substances. Nor are all the successes confined to the relatively simple chemical compounds-the theory of resonance in conjugated compounds has had great influence on the theories of the stability and reactivity of organic molecules.

Another field in which the appearance of quantum concepts has led to new understanding and fresh impetus is that of chemical reaction kinetics. In this book the basis of the work of Eyring and his school on one hand and of Polanyi on the other is very fully discussed. One chapter is devoted to the application of these conceptions to the semiempirical calculation of activation energies and to the calculation of absolute reaction velocities.

"Einführung in die Quantenchemie" is filling a real need. For those who are able to use the mathematics of the approximate methods of quantum chemistry, this monograph with its very full bibliography is an admirable introduction. On the other hand, by collecting in one place and correlating the various methods which have been used in quantum chemistry, this book may enable readers to find a conceptual structure which will give rise to a new co-ordination of chemical phenomena in a form which can be understood and handled by non-mathematicians.

\title{
Mathematics for Technical Students
}

\section{(I) Calculus}

By Prof. Herman W. March and Prof. Henry C. Wolff. (Modern Mathematical Texts.) Pp. xvii + 424. (New York and London: McGraw-Hill Book Co., Inc., 1937.) $15 s$.

\section{(2) The Elementary Theory of Operational} Mathematics

By Prof. Eugene Stephens. (Electrical Engineering Texts.) Pp. xi + 313. (New York and London : McGraw-Hill Book Co., Inc., 1937.) $21 s$.

(1) "T THE bond of union among the physical sciences is the mathematical spirit and the mathematical method which pervade them." Thus in their admirable text-book, Profs. March and Wolff endeavour to develop the fundamental principles of the calculus which play so important a part in the physical sciences, and to lay the emphasis upon the mode of thought so that even though the student may forget the details of the subject, he will continue to apply these fundamental modes of thinking in his later scientific or technical career.

While the needs of technical students have been foremost in the minds of the authors, the book is equally adapted to others. For, in the opinion of Profs. March and Wolff, mathematical courses for technical students and for the students of pure science do not materially differ. Both classes of students profit by the type of study that is assumed to be adapted to the other class.

The book is not divided into two separate parts devoted to the differential and integral calculus respectively. Integration with the determination of the constant of integration and the definite integral as the limit of a sum, are given immediately following the differentiation of algebraic functions, and before the differentiation of the transcendental functions. Thus many of the most important applications of the calculus occur early in the course and constantly recur. Further, by this arrangement the student is in a better position to pursue courses in physics and mechanics simultaneously with the calculus.

(2) In electrical engineering, the algebraic method as an aid to the simplification of work with the differential equations and integrals encountered in mathematical physics, has only been partially appreciated hitherto. The text of Prof. Stephens's book on the elementary theory of operational mathematics is an attempt both to search out the history of these methods and to codify the set of theorems found. Owing to the fundamental character of the methods used, the book will probably be of interest to the mathematician; but the author is careful to point out that it is written primarily for "all those who live and work to make mathematics useful to mankind, because of the beauty and simplicity of the operational forms and because of their wide application to the daily tasks of teachers and workers in engineering schools and industrial laboratories".

The essential nature of the method here presented consists of : (1) 'algebraizing' the operators of the differential and integral calculus; (2) simplifying the algebraic forms thus obtained; and (3) re-interpreting the resulting forms as operators which are then found to be easier to use than the original ones. Wherever the methods are not rigorously justified, reference is made to "The Theory of Linear Operators", by H. T. Davis ; and a very extensive bibliography is provided. 